\title{
Efeito da Endogamia sobre Características Produtivas e Reprodutivas de Bovinos do Ecótipo Mantiqueira1
}

\section{Marcos Vinícius Gualberto Barbosa da Silva ${ }^{2,3}$ William José Ferreira ${ }^{3}$, Jaime Araujo Cobuci ${ }^{3}$, Guilherme Paes Guaragna2, Paulo Rogério Palma de Oliveira²}

\begin{abstract}
RESUMO - Foram analisados registros de genealogia de 2070 animais, bem como informações provenientes das cinco primeiras lactações de 1406 vacas do ecótipo Mantiqueira, filhas de 113 reprodutores, com o objetivo de avaliar a freqüência de animais endogâmicos, a média de endogamia por geração e o possível reflexo do aumento da endogamia sobre algumas características reprodutivas e produtivas. As características avaliadas foram idade ao primeiro parto (IPP), intervalo de partos (IDP), produção total de leite (PL) e duração da lactação (DL). Foram usados modelos que incluíam, para PL, IDP e DL, os efeitos fixos de ano-estação de parto; como covariável, a idade da vaca ao parto, em meses, com termos linear e quadrático, além dos efeitos aleatórios de animal, de ambiente permanente e erro. Na análise envolvendo a PL, incluiu-se ainda o efeito fixo da duração da lactação. No estudo de IPP, o modelo utilizado considerou como efeito fixo ano-estação de nascimento e, como aleatórios, animal e erro. As análises foram realizadas utilizando-se a metodologia da máxima verossimilhança restrita. Para se verificar o comportamento da endogamia ao longo dos anos, os animais foram agrupados em gerações. Verificou-se aumento expressivo do número de animais endogâmicos, bem como dos níveis médios do coeficiente de endogamia, que oscilaram entre 0,33 e 7,34\%, com crescimento até a última geração. Observaram-se efeitos linear da endogamia sobre os valores genéticos para PL e IPP e quadrático para IDP e DL. A endogamia influenciou de maneira negativa os valores genéticos para as características estudadas, promovendo reduções para PL e DL e aumentos para IPP e IDP. A falta de um programa de acasalamento eficiente e, principalmente, o fato de o rebanho ser fechado, têm sido fatores determinantes no aumento contínuo do nível de endogamia e do número de animais endogâmicos.
\end{abstract}

Palavras-chave: bovinos de leite, endogamia, ecótipo Mantiqueira, metodologia REML, modelo animal

\section{Effect of the Inbreeding on Reproductive and Productive Traits in Mantiqueira Type Cattle}

\begin{abstract}
Genealogies of 2070 animals and records from the first five lactations of 1406 cows of the Mantiqueira type cattle, daughters of 113 sires, were analyzed to estimate the frequency of inbred animals, the inbreeding average per generation and the possible effect of inbreeding increase on reproductive and productive traits. The evaluated traits were age at first calving (IPP), calving intervals (IDP), total milk production (PL) and lactation period (DL). Models for PL, IDP and DL included the fixed effects of year-season of calving were used, linear and quadratic effects of the age of the cow as covariate and the random animal effects, permanent environment and error. The fixed effect of lactation period was included also in the PL analysis. Year-season of calving as fixed, and animal and error as random were fit in the mode for IPP. Restricted maximum likelihood methodology was used in the analyses. Trends of inbreeding were estimated by generation. Significant increases of inbred animals and average levels of inbreeding coefficient were observed with the latter varying between 0.33 and $7.34 \%$ and increasing up to the last generation. Linear effects of inbreeding on breeding values for PL and IPP and quadratic effect for IDP and DL were found. Inbreeding influenced negatively the breeding values for all traits being responsible for reductions in PL and DL and increases in IPP and IDP. Poor efficient breeding programs and the fact that the herd is closed are important factors for the continuous increase in the level of inbreeding and number of inbred animals.
\end{abstract}

Key Words: animal model, dairy cattle, inbreeding, Mantiqueira type, REML methodology

\section{Introdução}

A criação do ecótipo Mantiqueira no Núcleo de Pesquisa Zootécnica Geraldo José Rodrigues Alckmin do Instituto de Zootecnia da Agência Paulista de Tecnologia dos Agronegócios da Secretaria de Agricultura e Abastecimento, do Estado de São Paulo
(NPZGJRA/IZ/APTA/SAA-SP), foi iniciada por volta do ano de 1952, ao ser adquirido um grupo de aproximadamente 50 animais, oriundos de rebanhos dos Estados de Minas Gerais e São Paulo. Os animais desse ecótipo, originado a partir do cruzamento entre a raça Holandesa e o gado crioulo da região da Serra da Mantiqueira, apresentavam, segundo GUARAGNA

\footnotetext{
1 INSTITUTO DE ZOOTECNIA - Parte integrante do projeto IZ - 14-001/52.

2 Pesquisadores Científicos - Núcleo de Pesquisa Zootécnica Geraldo J.R. Alckmin/IZ/APTA/SAA CP 07 - Pindamonhangaba - SP.

3 Alunos de Pós-graduação, DZO/UFV. E.mail: mbarbosa@tdnet.com.br
} 
(1986), características morfológicas bem definidas, boa produção de leite e adaptação às propriedades de baixo nível tecnológico.

Desde a formação do rebanho até o início da década de 70, procurou-se simplesmente aumentar o plantel, sem preocupação com acasalamentos endogâmicos. Nesse período, de modo análogo ao descrito por QUEIROZ et al. (2000), a endogamia era utilizada juntamente com a seleção, de modo a assegurar a uniformidade racial e fixação de certas características.

Somente em 1974, de acordo com GUARAGNA et al. (1984), foi elaborado um plano de acasalamento entre animais não aparentados, de modo a evitar taxas elevadas de endogamia. Como consequiências dessas taxas elevadas, pode-se ter perda parcial do ganho genético obtido por seleção e a redução do valor médio fenotípico, evidenciado, principalmente, pelos caracteres relacionados à capacidade reprodutiva ou eficiência fisiológica, fenômeno este conhecido por depressão endogâmica (FALCONER e MACKEY, 1996 e WEIGEL e LIN, 2000). Além disso, segundo ALCALÁ et al. (1995), taxas de endogamia superiores a $10 \%$ podem ser perigosas, pois aumentam o número de locos em homozigose, evidenciando genes recessivos indesejáveis.

A endogamia, segundo OLIVEIRA et al. (1999), é um processo difícil de ser evitado em populações fechadas, particularmente naquelas na qual a seleção é praticada em apenas uma característica. Em estudos sobre endogamia em gado leiteiro, HUDSON e VAN VLECK (1984) mencionaram que sua ação pode reduzir a produção de leite e gordura, aumentar a taxa de mortalidade de bezerros, bem como influenciar adversamente na habilidade reprodutiva de novilhas e vacas. Tal fato tambémfoi observado por SMITH et al. (1998), que relataram diminuições nas produções de leite, gordurae proteína de, respectivamente, 37,2, 1,2 e 1,2 kg para cada acréscimo de $1 \%$ de endogamia. Estes mesmos autores constataram aumentos de 5,0 e 3,3 dias, respectivamente, na idade ao primeiro parto e primeiro intervalo de partos, em taxas superiores a $12,5 \%$ de endogamia.

Decréscimos em características produtivas, para cada incremento de $1 \%$ na endogamia, foram também observados por WIGGANS et al. (1995), os quais verificaram reduções nas produções de leite, gordura e proteína, por lactação de, respectivamente, 29,6,1,1 e 1,0 kg, para raça Holandesa, e de 21,3, 1,0 e $0,9 \mathrm{~kg}$, para raça Jersey. Além disso, THOMPSON et al. (2000a) verificaram que níveis superiores a $10 \%$ de endogamia influenciaram significativamente a duração da lactação, reduzindo-a em até 8 dias.

Assim, objetivou-se com este estudo avaliar a freqüência de animais endogâmicos, a média de endogamia por geração, bem como o possível reflexo do aumento da endogamia em algumas características reprodutivas e produtivas do ecótipo Mantiqueira, mantido no NPZGJRA/IZ/APTA/SAA-SP.

\section{Material e Métodos}

Os dados para este trabalho foram obtidos dos livros de escrituração zootécnica do NPZGJRA/IZ/ APTA/SAA-SP, desde a formação do rebanho Mantiqueira, na década de 50. Foram incluídos somente registros de vacas com um ou mais partos e de bezerras e novilhas para futura reposição no rebanho, assim como de todos os machos selecionados como reprodutores, totalizando 2.070 animais.

O núcleo básico foi formado pela aquisição de vacas e touros de quatro rebanhos de Minas Gerais e de um rebanho de São Paulo e, desde então, o rebanho foi fechado, não sendo mais permitida a introdução de material genético de qualquer origem. Da formação do rebanho até o ano de 1974, simplesmente procurou-se ampliar o número de animais, sem que houvesse um programa de acasalamento entre animais não aparentados. Apenas em 1974, elaborou-se tal programa, utilizando-se quatro touros de origens distintas ("linhagens") e tendo como critério a não existência de avós em comum, o que foi feito até 1986 , quando foram criadas mais seis "linhagens". Desde 1986, são selecionados, anualmente, dez reprodutores (um de cada "linhagem"), com base na primeira lactação de suas mães, desenvolvimento ponderal e tipo. Quanto às fêmeas, todas as novilhas eram incorporadas ao rebanho, desde que não tivessem problemas reprodutivos ou defeitos anatômicos. Ressalta-se que o direcionamento dos acasalamentos sempre foi feito mediante inspeção visual dos livros de escrituração zootécnica.

Os coeficientes de endogamia e valores genéticos de cada indivíduo, para as características produção total de leite (PL), idade ao primeiro parto (IPP), intervalo de partos (IDP) e duração da lactação (DL), foram obtidos por meio do sistema MTDFREML (BOLDMAN et al., 1995). Os regis- 
1238 Rev. bras. zootec.

tros utilizados, provenientes das cinco primeiras lactações de 1406 vacas do ecótipo Mantiqueira, filhas de 113 reprodutores, foram analisados por meio de modelos (modelo animal) que incluíam, para as características PL, IDP e DL, os efeitos fixos de ano-estação de parto (águas: outubro a março e seca: abril a setembro); como covariável, a idade da vaca ao parto, em meses, com termos linear e quadrático, além dos efeitos aleatórios de animal, de ambiente permanente e erro. $\mathrm{Na}$ análise envolvendo a PL, incluiu-se, ainda, o efeito fixo da duração da lactação. No modelo utilizado para o estudo da IPP, considerou-se como efeito fixo o ano-estação de nascimento e como aleatórios, animal e o erro.

Para se verificar o comportamento da endogamia ao longo dos anos, os animais foram agrupados em gerações. A geração de cada animal foi definida de acordo com o coeficiente proposto por BRINKS et al. (1961):

$\mathrm{CGI}=(\mathrm{CGP}+\mathrm{CGM}+2) / 2$, em que:

CGI = coeficiente de geração do indivíduo;

CGP = coeficiente de geração do pai; e

CGM = coeficiente de geração da mãe.

Foram considerados pertencentes à geração zero (população base) os animais fundadores, os incorporados ao rebanho e os de genealogia desconhecida.

Para se verificar a influência da endogamia sobre os valores genéticos dos animais, para as características reprodutivas e produtivas, foram realizadas análises de regressão. Para isso, os animais foram agrupados em classes de endogamia, conforme descrito na Tabela 1.

Tabela 1 - Número de animais por classe de endogamia Table 1 - Number of animals by inbreeding class

\begin{tabular}{lcc}
\hline $\begin{array}{l}\text { Coeficiente de } \\
\text { endogamia } \\
\text { Inbreeding } \\
\text { coefficient }\end{array}$ & $\begin{array}{c}\text { Classe de } \\
\text { endogamia } \\
\text { Inbreeding } \\
\text { class }\end{array}$ & $\begin{array}{c}\text { Número de } \\
\text { animais } \\
\text { Number of } \\
\text { animals }\end{array}$ \\
\hline$<0,010$ & 0 & 646 \\
0,010 a 0,029 & 2 & 179 \\
0,030 a 0,049 & 4 & 379 \\
0,050 a 0,069 & 6 & 366 \\
0,070 a 0,089 & 8 & 294 \\
0,090 a 0,109 & 10 & 96 \\
0,110 a 0,129 & 12 & 31 \\
0,130 a 0,149 & 14 & 37 \\
0,150 a 0,189 & 18 & 25 \\
$\geq 0,190$ & 22 & 17 \\
\hline
\end{tabular}

\section{Resultados e Discussão}

As estatísticas descritivas do coeficiente de endogamia $(\mathrm{F})$ para touros e vacas nascidos entre os anos de 1952 e 1997 encontram-se na Tabela 2. A média de $\mathrm{F}$ dos touros foi inferior à das vacas, o que pode ser explicado pela não utilização dos valores genéticos dos machos como critério de seleção. De acordo com MIGLIOR e BURNSIDE (1995), a intensa utilização de touros com valores genéticos mais elevados para produção de leite e de seus componentes tende a aumentar os laços genéticos entre os reprodutores.

Observa-se ainda, na Tabela 2, que de 1424 animais pertencentes ao rebanho, aproximadamente $69 \%$ eram endogâmicos, com coeficiente de endogamia médio de 5,9\%. Este valor está próximo a 6,25\%, que, segundo FRANCK (1997), é considerado, por muitos autores, como o nível máximo, a partir do qual haveria comprometimento dos desempenhos reprodutivo e produtivo dos animais. De acordo com THOMPSON et al. (2000a), reduções de até $55 \mathrm{~kg}$ na produção de leite por lactação estão relacionadas a níveis de endogamia superiores a 7,0\%.

$\mathrm{Na}$ Tabela 3 , pode ser verificado que houve aumento gradativo da endogamia dos touros e das vacas, ao longo das gerações, fato que, segundo QUEIROZ et al. (2000), pode ser explicado pela própria estrutura dos rebanhos, cujos animais fundadores são pouco aparentados ou de genealogia desconhecida e, com o passar do tempo, mediante a ocorrência de acasalamentos dentro do rebanho,

Tabela 2 - Descrição dos animais estudados do ecótipo Mantiqueira, quanto ao coeficente de endogamia $(F)$

Table 2 - Description of mantiqueira cattle, according to inbreeding coefficient $(F)$

\begin{tabular}{|c|c|c|c|}
\hline & $\begin{array}{l}\text { Vacas } \\
\text { Cows }\end{array}$ & $\begin{array}{l}\text { Touros } \\
\text { Bulls }\end{array}$ & $\begin{array}{l}\text { Total } \\
\text { Total }\end{array}$ \\
\hline Número total & 1920 & 150 & 2070 \\
\hline $\begin{array}{l}\text { Total number } \\
\% \text { de animais } \\
\text { endogâmicos } \\
\% \text { of inbred animals }\end{array}$ & 69,2 & 64,0 & 68,8 \\
\hline $\begin{array}{l}\text { F médio da população }(\% \\
\text { Average Population } F(\%)\end{array}$ & b) 4,1 & 3,6 & 4,0 \\
\hline $\begin{array}{l}\text { F médio de animais } \\
\text { endogâmicos }(\%) \\
\text { Average F of inbred } \\
\text { animals (\%) }\end{array}$ & 5,9 & 5,6 & 5,9 \\
\hline $\begin{array}{l}\text { Amplitude de } \mathrm{F}(\%) \\
\text { Range of } F(\%)\end{array}$ & $0,0-27,0$ & $0,0-15,0$ & $0,0-27,0$ \\
\hline
\end{tabular}


SILVA et al.

Tabela 3 - Coeficiente de endogamia (\%) para vacas, touros e total por geração

Table 3 - Inbreeding coeffcient (\%) for cows, sires and overall, per generation

\begin{tabular}{lccc}
\hline $\begin{array}{l}\text { Geração } \\
\text { Generation }\end{array}$ & $\begin{array}{c}\text { Vacas } \\
\text { Cows }\end{array}$ & $\begin{array}{c}\text { Touros } \\
\text { Bulls }\end{array}$ & $\begin{array}{c}\text { Total } \\
\text { Total }\end{array}$ \\
\hline 0 & 0,00 & 0,00 & 0,00 \\
1 & 0,00 & 0,00 & 0,00 \\
2 & 0,34 & 0,00 & 0,33 \\
3 & 0,74 & 0,68 & 0,73 \\
4 & 2,78 & 2,47 & 2,76 \\
5 & 4,48 & 4,79 & 4,50 \\
6 & 5,67 & 6,15 & 5,71 \\
7 & 5,62 & 5,43 & 5,61 \\
8 & 6,56 & 6,69 & 6,56 \\
9 & 7,34 & - & 7,34 \\
\hline
\end{tabular}

aumenta o grau de parentesco entre os indivíduos, o que leva à endogamia. Na geração 8 , o valor médio ultrapassou o limite de $6,25 \%$, chegando a $7,34 \%$ na geração 9 , indicando que nestas últimas gerações o aumento foi próximo a $1 \%$. A partir da geração 6 (Figura 1), a totalidade dos animais nascidos no rebanho era endogâmica, evidenciando que, a curto prazo, os efeitos da depressão endogâmica podem ser acentuados, ocasionando perda da variabilidade genética, redução da produção de leite bem como da eficiência reprodutiva em geral.

Concordando com os resultados encontrados por PENNA (1990), a partir do nível de $10 \%$ de endogamia, houve tendência de aumento nos valores genéticos para IDP (Figura 2), com ponto de mínimo para

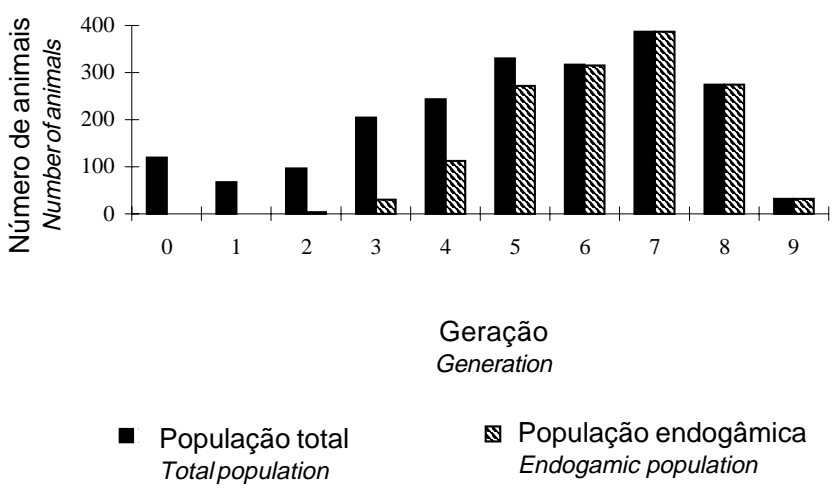

Figura 1 - Número total de animais endogâmicos ao longo das gerações.

Figure 1 - Total number of inbreeding animals per generation.
$\mathrm{F}=10,86 \%$. Para IPP, observou-se tendência linear de aumento de 0,12 meses nos valores genéticos, para cada acréscimo de $1 \%$ no coeficiente de endogamia (Figura 2). Influência significativa da endogamia sobre IPP também foi relatada por THOMPSON et al. (2000a, 2000b), os quais encontraram aumentos de até 17 e 12 dias para IPP, para níveis superiores a $10 \%$ de endogamia, nas raças Holandesa e Jersey, respectivamente.

Conforme pode ser verificado na Figura 3, até $10 \%$ a endogamia não influenciou nas classificações das vacas quanto ao valor genético para IPP. Todavia, a partir desse nível, os animais com os maiores coeficiente de endogamia eram os que possuíam maiores valores genéticos para IPP, levando-os a ocupar as últimas posições. Tendência semelhante foi observada para IDP.

Na Figura 4 encontram-se as regressões dos valores genéticos para PL e DL, em função das classes de endogamia. Para níveis crescentes de endogamia observa-se tendência linear de diminuição dos valores genéticos para produção de leite. A incrementos de $1 \%$ no coeficiente de endogamia correspondem redução de, aproximadamente, $1,15 \mathrm{~kg}$ de leite. Efeitos da endogamia sobre a produção de leite também foram constatados por HUDSON e VAN VLECK (1984), SMITH et al. (1998) e THOMPSON et al. (2000a e 2000b). Em relação a DL, tem-se comportamento quadrático dos valores genéticos em relação aos níveis de endogamia, com ponto de máximo para $\mathrm{F}=11,44 \%$. Até este ponto, houve tendência de aumentos do valores genéticos e,

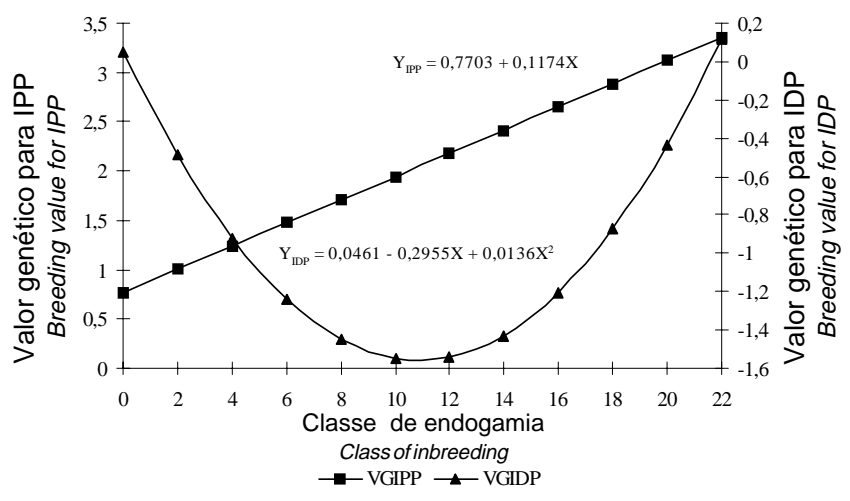

Figura 2 - Valores genéticos para idade ao primeiro parto (VGIPP) e intervalo de partos (VGIDP), em meses e dias, respectivamente, de acordo com a classe de endogamia.

Figure 2 - Breeding values for age at first calving (VGIPP) and for calving interval (VGIDP), in months and days, respectively, by inbreeding class. 
1240 Rev. bras. zootec.

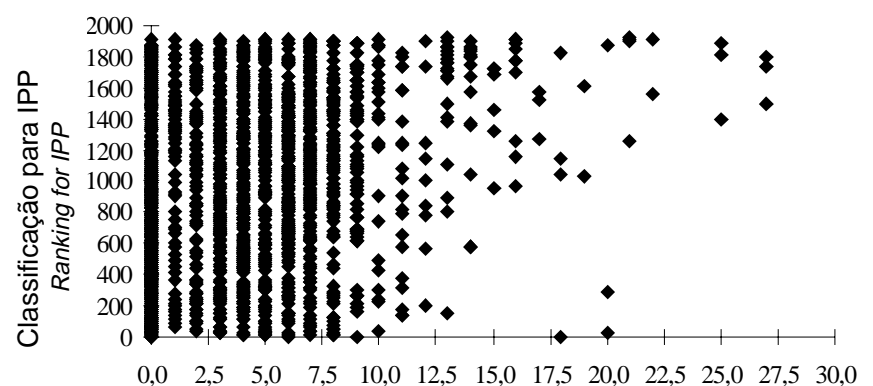

Coeficiente de endogamia (\%) INbreeding coeficient (\%)

Figura 3 - Dispersão das classificações de vacas quanto aos seus valores genéticos para idade ao primeiro parto (IPP) e coeficiente de endogamia (\%).

Figure 3 - Plot of cows rankings, according to breeding values for age at first calving (IPP) and inbreeding coefficient (\%).

a partir daí, a endogamia influenciou de forma negativa, isto é, reduzindo os valores genéticos dos animais para essa característica. THOMPSON et al. (2000a e 2000b) observaram, para níveis superiores a $5 \%$ de endogamia, decréscimo de até 8 e 5 dias, respectivamente, na DL.

De modo análogo ao observado para IPP, até o nível de $10 \%$, as classificações das vacas quanto ao valor genético paraPLnãoforaminfluenciadas pelaendogamia, entretanto, a partir desse nível, os animais com os maiores coeficiente de endogamia foram aqueles que apresentaram menores valores genéticos para PL (Figura 5). Resultado similar foi encontrado para DL.

Como o efeito da endogamia sobre os valores genéticos para PL e IPP foi linear, pode-se inferir que os locos que expressam essas características combinam-se aditivamente. Entretanto, para IDP e DL, o comportamento quadrático sugere que a interação epistática entre locos é importante (FALCONER e MACKEY, 1996).

Por meio de um estudo comparativo entre o incremento real (DF real) de endogamia e o incremento esperado, caso os acasalamentos fossem realizados aleatoriamente (DF teórico), concluiu-se que, com exceção das gerações 1 e 2 , em que o número de machos é reduzido em relação às demais gerações, o DF teórico se mantém constante, enquanto o DF real aumenta até a quarta geração, diminuindo a partir daí (Tabela 4). Tal comportamento, provavelmente, pode ser explicado pela utilização de um critério de acasalamento de animais não aparentados, iniciado em 1974 e coincidente com o nascimento dos animais

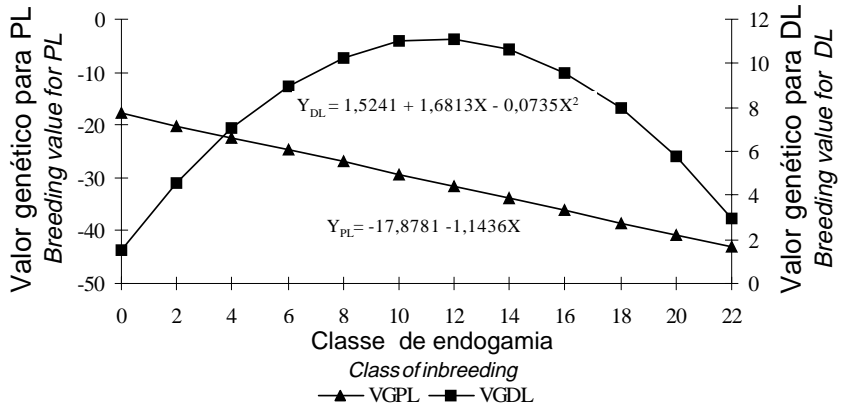

Figura 4 - Valores genéticos para produção de leite (VGPL) e para duração da lactação (VGDL), em kg e dias, respectivamente, de acordo com a classe de endogamia.

Figure 4 - Breeding values for milk yield (VGPL) and for lactation lenght (VGDL), in kilograms and days, respectively, per inbreeding class.

pertencentes a geração 5. Destaca-se que, na geração 9 , o valor do DF real é, aproximadamente, 1/3 do valor obtido na geração 4 , o que demonstra alguma melhoria em relação ao período no qual o parentesco entre os indivíduos, pertencentes à população, não era considerado nos acasalamentos (gerações 0 a 4 ).

Deve ser considerado que a endogamia influencia as características de crescimento e reprodutivas.

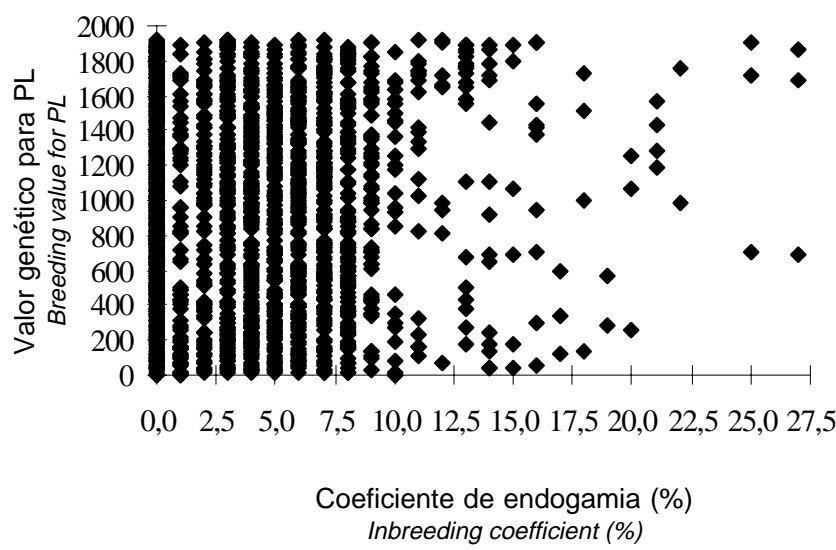

Figura 5 - Dispersão das classificações de vacas quanto aos seus valores genéticos para produção de leite (PL) e coeficiente de endogamia (\%).

Figure 5 - Plot of cows rankings, according to breeding values for milk yield (PL) and inbreeding coefficient (\%). 
SILVA et al.

Tabela 4 - Comparação dos coeficientes de endogamia teóricos com os reais Table 4 - Comparison between theoretical and actual inbreeding coefficients

\begin{tabular}{lcccccc}
\hline $\begin{array}{l}\text { Coeficiente de geração } \\
\text { Coefficient of generation }\end{array}$ & $\mathrm{Nm}$ & $\mathrm{Nf}$ & $\begin{array}{c}\text { DF teórico } \\
\text { DF theoretical }\end{array}$ & $\begin{array}{c}\text { F acumulado } \\
\text { Acumulated } F\end{array}$ & $\begin{array}{c}\text { F médio/geração } \\
\text { Faverage/generation }\end{array}$ & $\begin{array}{c}\text { DF real } \\
\text { DF actual }\end{array}$ \\
\hline 0 & 23 & 96 & 0,674 & 0,674 & 0,00 & 0,00 \\
1 & 6 & 61 & 2,288 & 2,962 & 0,00 & 0,00 \\
2 & 4 & 93 & 3,259 & 6,221 & 0,33 & 0,73 \\
3 & 16 & 189 & 0,847 & 7,068 & 2,76 & 0,40 \\
4 & 15 & 228 & 0,888 & 7,956 & 5,50 & 2,03 \\
5 & 19 & 311 & 0,698 & 8,654 & 5,71 & 1,74 \\
6 & 26 & 291 & 0,524 & 9,178 & 6,61 & $-0,10$ \\
7 & 28 & 358 & 0,481 & 9,659 & 7,34 & 0,95 \\
8 & 13 & 262 & 1,009 & 10,668 & - & 0,78 \\
$9 *$ & - & 31 & - & $-3,34$ & \\
\hline
\end{tabular}

* A geração 9 não foi considerada no cálculo do DF teórico, pois não havia machos nesta geração (The generation number 9 was not considered to estimate DF theoretical, because there were no males); $\mathrm{Nm}=$ número de machos envolvidos na formação de cada geração; (Nm = number of males involved in the formation of each generation); $\mathrm{Nf}=$ número de fêmeas envolvidas na formação de cada geração; (Nf = number of females involved in the formation of each generation); DF teórico $=[1 /(8 \mathrm{Nm})+1 /(8 \mathrm{Nf})] ;(D F$ theoretical $=[1 /\{8 \mathrm{Nm}\}+1 /\{8 \mathrm{~N} f])$; DF real = $\mathrm{F}$ médio real da geração anterior $-\mathrm{F}$ médio da geração posterior; (DF actual = average of $F$ in the next generation - average of $F$ in the previous generation).

Dessa forma, pode-se inferir que a endogamia tenha, possivelmente, maior ação que a verificada neste estudo, uma vez que indivíduos altamente endogâmicos podem ter sido descartados pelo baixo peso ao nascimento, desenvolvimento ponderal inadequado e por problemas reprodutivos e, por isso, não constavam nos registros analisados.

De acordo com MIGLIOR e BURNSIDE (1995), em programas de melhoramento, espera-se o aumento da endogamia em gado de leite, resultante de sua própria estrutura, como por exemplo: alta intensidade de seleção, uso de técnicas reprodutivas, avaliações genéticas mais precisas e uso intenso de alguns reprodutores. Além disso, a seleção com base nos valores genéticos estimados pode aumentara endogamia, uma vez haverá parentesco entre os animais de maior mérito genético. Especificamente, para o rebanho estudado, tem-se o agravante de não haver introdução de material genético proveniente de outros rebanhos, acelerando o processo de aumento da endogamia.

\section{Conclusões}

Ocorreu aumento do coeficiente de endogamia e do número de animais endogâmicos ao longo das gerações, sendo que a partir da sexta geração todos os animais eram endogâmicos.

A endogamia influenciou de maneira negativa os valores genéticos para as características estudadas, promovendo reduções para produção de leite e duração da lactação e aumentos para idade ao primeiro parto e intervalo de partos.

A falta de um programa de acasalamento eficiente e, principalmente, o fato de o rebanho ser fechado têm sido fatores determinantes no crescimento contínuo do nível de endogamia e do número de animais endogâmicos. Assim, a médio prazo, as reduções observadas na fertilidade poderão ser acentuadas.

\section{Referências Bibliográficas}

AlCAlÁ, A.M., FRANGANILlO, A.R., CÓRDOBA, M.V. 1995. Analisis genetico de los niveles de consanguinidad en la raza Retinta. Arch. Zootec., 44(166-167):257-265.

BOLDMAN, K.G., KRIESE, L.A., VAN VLECK, L.D. et al. 1995. A manual for use of MTDFREML. A set of programs to obtain estimates of variances and covariances. [DRAFT] Beltsville: U.S. Department of Agriculture, Agricultural Research Service. $125 \mathrm{p}$.

BRINKS, J.W., CLARK, R.T., RICE, F.J. 1961. Estimation of genetic trends in beef cattle. J. Anim. Sci., 20(4):903.

FALCONER D.S., MACKAY, T.F.C. 1996. Introduction to Quantitative Genetics. 4.ed. New York: Longman Group Limited. 464p.

FRANCK, R. 1997. Inbreeding steals profits. Dairy Herd Management. p.46-50.

GUARAGNA, G.P. Reprodução e endogamia em bovinos Mantiqueira. Ribeirão Preto, SP:FMRP, 1986. 136 p. Tese (Doutorado em Genética) - Faculdade de Medicina de Ribeirão Preto/Universidade de São Paulo,1986.

GUARAGNA, G.P., GAMBINI, L.B., FIGUEIREDO, A.L. 1984 Mantiqueira: 30 anos depois. Nova Odessa: Instituto deZootecnia, Secretaria de Agricultura e Abastecimento de São Paulo. 30p. 
1242 Rev. bras. zootec.

HUDSON, G.F.S., VAN VLECK, L.D. 1984. Effects of inbreeding on milk and fat production, stayability, and calving interval of registered Ayshire catlle in the northeastern United States. J. Dairy Sci., 67(1):171-179.

MIGLIOR, F., BURNSIDE, E. B. 1995. Inbreeding of Canadian Holstein cattle. J. Dairy Sci., 78(5):1163-1167.

OLIVEIRA, J.A., BASTOS, J.F.P., TONHATI, H. 1999. Endogamia em um rebanho da raça Guzerá. Rev. bras. zootec., 28(4):721-728.

PENNA, V.M. Efeito da endogamia em características de peso e reprodução da raça Tabapuã. Ribeirão Preto, SP:FMRP, 1990. 102 p. Tese (Doutorado em Genética) - Faculdade de Medicina de Ribeirão Preto/Universidade de São Paulo, 1990.

QUEIROZ, S.A., ALBUQUERQUE, L.G., LANZONI, N.A. 2000. Efeito da endogamia sobre características de crescimento de bovinos da raça Gir no Brasil. Rev. bras. zootec., 29(4):1014-1019.
SMITH, L.A., CASSELL, B.G., PEARSON, R.E. 1998. The effects of inbreeding on the lifetime performance of dairy cattle. J. Dairy Sci., 81(10):2729-2737.

THOMPSON, J.R., EVERETT, R.W., HAMMERSCHMIDT, N.L. 2000a. Effects of inbreeding on production and survival in Holsteins. J. Dairy Sci., 83(8):1856-1864.

THOMPSON, J.R., EVERETT, R.W., WOLFE, C.W. $2000 \mathrm{~b}$. Effects of inbreeding on production and survival in Jerseys. J. Dairy Sci., 83(9):2131-2138.

WEIGEL, K.A., LIN, W. 2000. Use of computerized mate selection programs to control inbreeding of Holstein and

Recebido em: $13 / 11 / 00$

Aceito em: 13/03/01 